\title{
Coding of Serial Order by Neostriatal Neurons: A "Natural Action" Approach to Movement Sequence
}

\author{
J. Wayne Aldridge ${ }^{1,2}$ and Kent C. Berridge ${ }^{2}$ \\ Departments of ${ }^{1}$ Neurology and ${ }^{2}$ Psychology, University of Michigan, Ann Arbor, MIchigan 48104-1687
}

The neostriatum controls behavioral sequencing, or action syntax, as well as simpler aspects of movement. Yet the precise nature of the neostriatums role in sequencing remains unclear. Here we used a "natural action" approach that combined electrophysiological and neuroethological techniques. We identified neostriatal neurons that code the serial order of natural movement sequences of rats. During grooming behavior, rats emit complex but highly predictable species-specific sequences of movements, termed "syntactic chains." Neuronal activity of $41 \%$ of cells in the dorsolateral and ventromedial neostriatum coded the sequential pattern of syntactic chains. Only $14 \%$ coded simple motor properties of grooming movements. Neurons fired preferentially during syntactic chains compared with similar grooming movements made in different sequential order or to behavioral resting. Sequential coding differed between the dorsolateral and ventromedial neostriatum. Neurons in the dorsolateral site increased firing by $116 \%$ during syntactic chains, compared with only a $30 \%$ increase by neurons in the ventromedial site, and dorsolateral neurons showed strongest coding of grooming syntax by several additional criteria. These data demonstrate that neostriatal neurons code abstract properties of serial order for natural movement and support the hypothesis that the dorsolateral neostriatum plays a special role in implementing action syntax.

Key words: movement sequences; basal ganglia; striatum; caudate nucleus; putamen; rat; neuronal activity; movement; syntax; grooming; fixed action pattern; Huntington's disease; Parkinson's disease; Tourette's; obsessive-compulsive disorder
What do the basal ganglia do for movement? In this paper we present evidence for one possible function: neostriatal neurons code the serial order of syntactic sequences of natural behavior. By syntactic we mean a sequence that follows rules imparting lawful predictability to the temporal progression of its elements. Although language is the ultimate example of syntax, other movement sequences besides speech can have syntax-like properties when their serial order is generated and controlled separately from individual movements (Lashley, 1951). Behavioral sequences can range from the complex skills of humans or learned motor sequences of animals to species-specific "instinctive" movement sequences. An example of a syntactic species-specific sequence is provided by grooming behavior of rats and other rodents. Grooming sequences follow predictable patterns (Fentress, 1972; Richmond and Sachs, 1978; Berridge et al., 1987), with the most stereotyped pattern linking up to 25 forelimb stroke and body lick movements into a four-phase "syntactic chain" of action that lasts $\sim 5 \mathrm{sec}$ (Berridge et al., 1987; Berridge, 1990).

The performance of syntactic grooming chains depends on the neostriatum. Ablative or excitotoxic lesions of the neostriatum disrupt the serial structure of the chain pattern, as do 6-hydroxydopamine lesions of nigrostriatal projection neurons (Berridge and Fentress, 1987; Berridge and Whishaw, 1992; Cromwell and Berridge, 1996). By contrast, lesions to other fore-

\footnotetext{
Received Oct. 15, 1997; revised Jan. 9, 1998; accepted Jan. 20, 1998.

The work was supported by National Institutes of Health Grant NS31650 (J.W.A.) and National Science Foundation Grant IBN9604408 (K.C.B.). We are grateful for able assistance: technical, J. F. Thompson and E. Laufenberg; computing, C. Chu and R. W. Kindt; hardware, D. Jones, T. Ryan, and I. Levin; and video scoring, E. Brasseur, L. Broyls, E. Butzer, J. Fletcher, A. Heath, S. Joshua, J. Kotler, E. Mayers, A. Memood, S. Raju, C. Sauber, S. Seth, J. Skalitsky, and E. Vadon.

Correspondence should be addressed to Dr. J. Wayne Aldridge, University of Michigan, 1103 East Huron, Neuroscience Building, Ann Arbor, MI 48104-1687.

Copyright (C) 1998 Society for Neuroscience $0270-6474 / 98 / 182777-11 \$ 05.00 / 0$
}

brain motor systems, including the primary and secondary motor cortex, the entire neocortex, or the cerebellum produce sensorimotor deficits in grooming movements but do not disrupt the syntactic pattern of grooming (Berridge and Whishaw, 1992). Recent work has demonstrated that the sequencing of grooming syntax is anatomically segregated within the neostriatum. Lesions as small as $1 \mathrm{~mm}$ in diameter will disrupt syntactic grooming if they fall within a crucial portion of the anterior dorsolateral striatum (Cromwell and Berridge, 1996). Thus the dorsolateral neostriatum of the rat plays an especially important role in movement sequencing.

Little is known, however, regarding the function contributed by neurons within the neostriatum for behavioral sequencing. Do neostriatal neurons trigger each successive movement, consistent with the generation of sequential patterns? Or do they instead code more abstract features of a sequential pattern, consistent with a role in pattern implementation? Neurophysiological investigations in primates performing trained movement sequences suggest that the neostriatum (Kermadi and Joseph, 1995; Miyachi et al., 1997) and pallidum (Mushiake and Strick, 1995) may play a vital role in motor sequence learning or performance. In a learned motor task, however, it is difficult to dissociate basic sequencing mechanisms that coordinate the serial order of a sequence from associative mechanisms for learning or remembering it. Natural grooming syntax has an advantage for dissociating movement sequencing from sequential memory, because lawful sequences are produced spontaneously without a need for training. A preliminary study (Aldridge et al., 1993) suggested that a population of neurons within the dorsolateral neostriatum might code the sequential pattern of rat syntactic grooming chains as a higher-order feature of motor control and separately from individual movements. 


\section{MATERIALS AND METHODS}

General. A syntactic grooming chain comprises four phases, each containing several movements of a particular type (see Fig. 3). Phase 1 consists of a series of five to nine rapid elliptical bilateral strokes over the nose and mystacial vibrissae lasting for $\sim 1 \mathrm{sec}$ (a bilateral movement made symmetrically by two paws is counted here as a single stroke even though it requires a movement by both limbs). Phase 2 consists of one or two small asymmetrical strokes of increasing amplitude lasting $<0.5 \mathrm{sec}$. Phase 3 consists of a series of three to six large bilateral strokes and lasts 2-3 sec. Phase 4 consists of a postural turn and head ducking followed by repeated body licking directed to the flank. The initiation of phase 4 completes the stereotyped syntax of the chain, although body licking may persist for up to $30 \mathrm{sec}$. For analytical purposes we categorized the period after the end of body licking as phase 5. Once a syntactic grooming chain begins, the remaining phases can be predicted with $\sim 90 \%$ accuracy. The entire syntactic chain of grooming movements occurs with a frequency $>13,000$ times greater than could be expected by chance (based on the relative probabilities of 25 grooming movements occurring in this order (Berridge et al., 1987). The four types of grooming action that compose the chain also occur outside of the syntactic chain sequence at even higher rates of occurrence in many "flexible" sequences of unpredictable order.

Animals. Sprague Dawley rats (250-400 gm) were used for these experiments and were housed on a $12 \mathrm{hr}$ light/dark schedule with lights out at 12 P.M. each day. All procedures were supervised and approved by University of Michigan Unit for Laboratory Animal Medicine and adhered to the NIH Guide for the Care and Use of Laboratory Animals, revised 1985.

Surgery. Aseptic surgical preparation occurred 4-7 d before recording. Animals were anesthetized with ketamine $(100 \mathrm{mg} / \mathrm{kg}$, i.p. $)$ and xylazine $(10 \mathrm{mg} / \mathrm{kg}$, i.p.) and placed in a stereotaxic frame. The skin and muscle were deflected, and a 2-mm-diameter section of bone was removed from the skull. We implanted multiple electrode arrays (eight tungsten microwires at $25 \mu \mathrm{m}$ each) based on a design from this laboratory (Jaeger et al., 1990). Electrodes were placed in either the dorsolateral neostriatum [centered around anteroposterior 0.2 and lateral (L) $3.5 \mathrm{~mm}$, with respect to bregma; 22 rats] or ventromedial neostriatum (AP -0.11 and L $2.2 \mathrm{~mm}$; nine rats). Neuronal activity was recorded during electrode implantation to ensure accurate placement relative to the boundaries of cortex, corpus callosum, and striatum. A circular array of bone screws around the implant was embedded in dental acrylic to secure the permanent electrode assembly.

Behavioral and neurophysiological recording. Rats were allowed to recover for 1 week before recording. Recording was done in the first few hours of the animal's dark (active) period in a darkened laboratory environment. Dim red light (reflected $25 \mathrm{~W}$ incandescent bulb), which does not alter the circadian rhythm, was used for videotaping with a low-light video camera. One week before surgery, each animal was handled on a daily basis and placed in the recording chamber and allowed to groom and explore to familiarize it to the testing environment and filming procedures.

During the recording session the animals were placed in a circular recording chamber $(30 \mathrm{~cm}$ diameter) over a clear plastic floor that allowed a video camera to record from below. A multiple-channel preamplifier and cable assembly (field effect transistor operational amplifier) was attached to a commutator to record neuronal activity, counterweighted to not impede movement by the rat. Grooming, walking, periods of quiet resting, and other movements were emitted spontaneously during $2 \mathrm{hr}$ sessions. Typically, each session included 10-15 syntactic chains. Neuronal activity and behavior were recorded continuously throughout the $2 \mathrm{hr}$ period. After long periods of quiescence, grooming was occasionally induced by spraying the fur with a fine mist of water. These testing procedures were essentially free of pain or distress for the rat.

Recorded single-neuron activity was amplified $(10,000 \times)$, filtered $(100-10,000 \mathrm{~Hz})$, and displayed on an oscilloscope while simultaneously monitored with an audio amplifier. Single-neuron activity was recorded by an on-line computer with a DataWave-based data acquisition system. Behavioral activity was videotaped simultaneously. To synchronize the neuronal and behavioral recordings, the same clock signal drove the time stamp clock in the computer recording neuronal activity and the behavioral time code recorded on each frame of the videotape.

Behavioral analysis. A frame-by-frame analysis of the videotapes was subsequently conducted off-line using both a choreographic notation system developed for detailed descriptions of stereotyped grooming sequences (Berridge and Fentress, 1986) and a computer-assisted scoring system (that transcribed the occurrence of each grooming stroke, lick, or other movement, as well as limb trajectory amplitude and laterality, and other movements such as rearing, stepping, head turning, and reaching). The computer extracted the time code from the frame and stored the information in a database along with the choreographic notation describing the movement. All syntactic grooming chains in the recording session were scored (6-17 chains per recording session; average, 9.5). Periods of nonchain grooming, resting, and other motor behavior were sampled throughout the recording session to accumulate, wherever possible, 10 events of each type for each neuron to be studied.

Neuronal activity analysis. Unit activity related to behavioral actions was assessed by standard perievent time histogram and raster techniques. Each perievent histogram was constructed around a behavioral event marking the onset of a sequential chain phase or a nonchain equivalent, using a computer program (Stranger, Biographics, Inc.). These alignment events included, for syntactic grooming chains, phase 1 onset (marked by onset of rapid elliptical strokes), phase 2 onset (marked by unilateral stroke after phase 1), phase 3 onset (marked by large bilateral strokes after phase 1 or 2), phase 4 onset (marked by body licking after phase 3 ), and the termination of phase 4 body licking (defined here as phase 5 in chains). For nonchain grooming, alignment events included rapid ellipselike strokes, unilateral strokes, large bilateral strokes, and body licking that were similar to chain components but that appeared in different serial order during ordinary grooming. Neuronal activation was assessed by visual inspection of the perievent histograms and rasters and by statistically evaluating population responses from portions of normalized histograms.

The intensity of phasic firing rate changes over short periods $(300$ $\mathrm{msec}$ ) within chains was determined by an analysis of the histograms of all neurons in the sample (responsive and nonresponsive) in 300 periods after the onset of each phase. For this analysis we normalized the changes for each neuron, first by computing the average rate from the onset of the phase until $300 \mathrm{msec}$ after the onset and then expressing this rate change as a percentage of the average rate in a baseline $1 \mathrm{sec}$ period, defined as -2 to $-1 \mathrm{sec}$ before the chain began. Behaviorally, this baseline period often included the terminal portion of a bout of nonchain grooming (because syntactic chains were usually embedded between bouts of nonchain grooming) or else behavioral rest. By ending the baseline $1 \mathrm{sec}$ before the onset of the chain, we ensured that any potential neuronal activity related to chain onset would not be included as part of a baseline (assuming that neuronal activity associated directly with the onset of muscle activity should occur at least $500 \mathrm{msec}$ before the movement). In this manner, the relative activation throughout each chain was normalized with respect to its own prechain baseline period to avoid spurious changes in firing rate from comparisons of dissimilar behavioral periods. These normalized percentage changes in firing rate for each neuron and phase were used for subsequent analyses.

In addition to the analysis of phasic spike activity changes to chain and nonchain events, we also examined and compared tonic neuronal activation properties during overall behavioral epochs (sequential chain grooming, flexible nonchain grooming, and behavioral rest). These epochs were delineated by behavioral event time markers identified in the frame-by-frame video analysis. Chain grooming epochs began $2 \mathrm{sec}$ before phase 1 (elliptical strokes) and ended $2 \mathrm{sec}$ after phase 4 (body licking). Nonchain grooming periods included all of the time spent grooming bounded by $2 \mathrm{sec}$ before the first grooming bout until $2 \mathrm{sec}$ after the last event in the bout (excluding any syntactic chain epochs) and averaged across all grooming bouts within a test session. Quiet resting behavior was demarcated in a similar manner surrounding periods when the animal remained in one place without moving for at least $15 \mathrm{sec}$ (average rest period duration was $\sim 5 \mathrm{~min}$ ). Average firing rates and the variability of interspike intervals (coefficient of variation) (Aldridge and Gilman, 1991) were compared across these behavioral epochs. Statistical evaluations were made with Systat (SPSS Inc.). All relationships that were tested statistically are indicated in the text or legends by the type of test and whether the outcome was significant $(p<0.05)$ or not significant $(p \geq 0.05)$.

Histology and localization. At the end of the recording session the location of the electrode was marked by passing a small lesioning current (100 $\mu \mathrm{A}$ for $20 \mathrm{sec}$ ) through the electrode tip that extended farthest along the array. The animals were killed with an overdose of pentobarbital and perfused intracardially with saline followed by $10 \%$ buffered formalin in $0.9 \%$ saline. Brains were removed from the skull, soaked in 10, 20, and $30 \%$ sucrose-formalin solutions, blocked, sectioned at $40 \mu \mathrm{m} / \mathrm{section}$, 


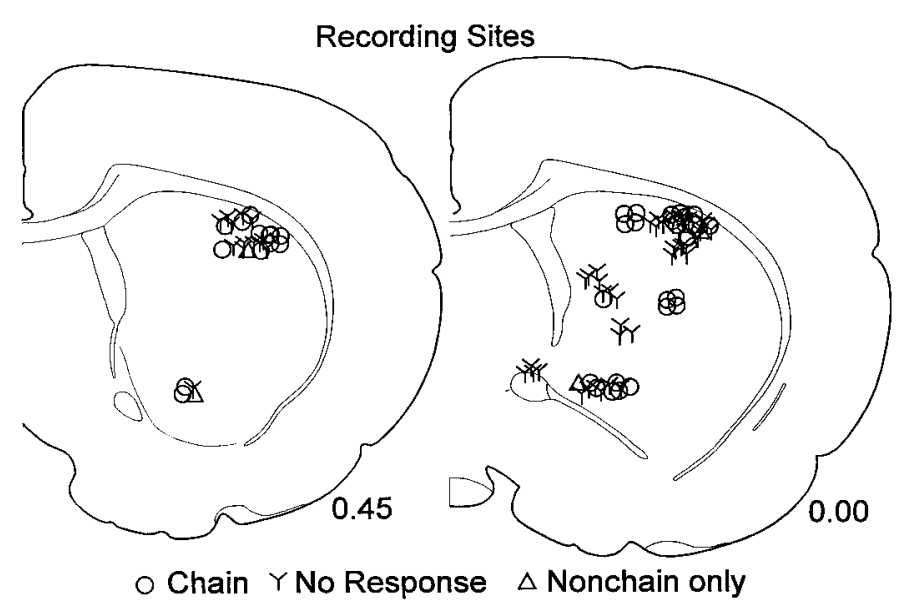

Figure 1. Recording sites in neostriatum. Each recorded cell is indicated as responsive to syntactic chains of grooming movements (circle), nonresponsive to any grooming movement $(Y$ symbol $)$ or responsive only to grooming movements occurring outside of syntactic chains (nonchain, triangle). Electrode recording sites from planes 0.5 to -0.1 referenced to bregma (Swanson, 1992) were plotted onto sections 0.45 or 0.00 (within $0.2 \mathrm{~mm}$ ) and dithered (for illustration) within a $0.25 \mathrm{~mm}$ radius around the lesion site. Because responsive and nonresponsive cells were recorded simultaneously, their anatomical locations were often adjacent, and so symbols overlap extensively.

and stained with cresyl violet. Recording sites (lesion marks) were identified and plotted on digitized maps from a standardized atlas (Swanson, 1992).

\section{RESULTS}

We compared neuronal activity across three behavioral contexts: syntactic grooming chains, ordinary nonchain grooming (which has similar forelimb stroke and lick movements but in different and unpredictable order), and quiet behavioral resting. A total of 116 neostriatal neurons in 31 animals were recorded and analyzed. Of the 116 neurons, 79 were confirmed to be within the 1-mm-diameter anterior dorsolateral site (Fig. 1) previously identified to be crucial for behavioral syntax (Cromwell and Berridge, 1996). An additional 37 neurons were in a ventromedial region of the neostriatum, in which lesions do not disrupt behavioral grooming syntax. Neurons from the two anatomical sites were explicitly compared in analyses to assess regional differences in function.

\section{Rate coding of behavioral epochs}

The average (median) firing rates were calculated separately for every neuron during the three types of behavioral epoch: syntactic grooming chains (average $80 \mathrm{sec} /$ neuron for all chains), other grooming bouts (368 sec/neuron), and behavioral resting (231 sec/neuron). Group comparisons revealed that the median firing rate differed significantly across these behavioral categories for neurons in the dorsolateral neostriatum (Fig. 2; Friedman twoway ANOVA, $p<0.001 ; n=50$ ). Chain epochs and flexible nonchain grooming epoch rates were both faster than behavioral resting rates (72 and $66 \%$, respectively; Wilcoxon signed rank test, $p<0.005$ and $p<0.001$, respectively; $n=50$ ). Furthermore, median firing rates during syntactic chain epochs were $16 \%$ faster than during nonchain grooming epochs (Wilcoxon test, $p<0.001$; $n=72$ ). The rate changes for dorsolateral neurons were not accompanied by detectable changes in variability (coefficient of variation) of interspike intervals (Kruskal-Wallis test, $p=0.06$ ) across behavioral states.
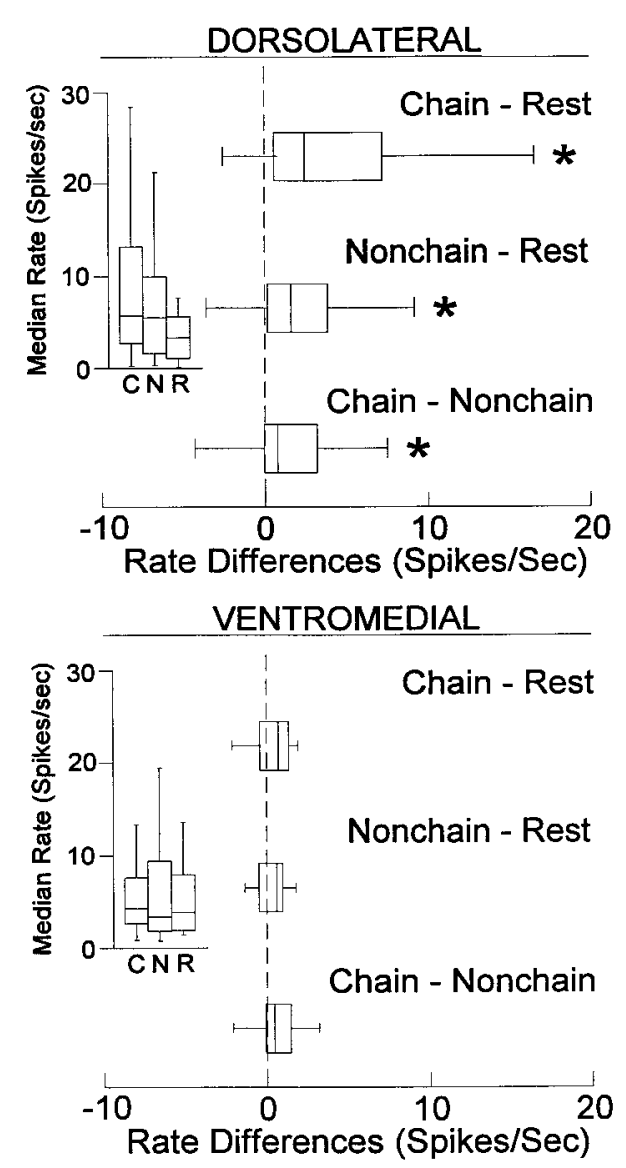

Figure 2. Coding of behavioral state by neurons in dorsolateral (top) and ventromedial (bottom) neostriatum. Median firing rates during syntactic grooming chains $(C)$, nonchain grooming $(N)$, and behavioral rest $(R)$ are shown by the vertical box plots in the insets at left (median rates were determined separately for each neuron). The differences between rates for each pair of behavioral states compared (Chain vs Rest, Nonchain vs Rest, and Chain vs Nonchain) are illustrated by the horizontal box plots. Dashed vertical line at zero indicates no difference in the two states being compared. Each box encompasses the central $50 \%$ of the sample $(25 \mathrm{th}-75$ th percentiles), the median value is indicated by the middle line in each box, and the whiskers extend 1.5 times the distance between the border of the box and the median value of the quadrant. Dorsolateral neurons differed significantly in absolute median firing rates (vertical bars, inset graph) across groups (Friedman two-way ANOVA, $p<0.001$ ). The withinneuron behavioral state comparisons (horizontal boxes) revealed a significant shift from zero toward positive values (one-sample $t$ test, $H_{0}=0$; Bonferroni adjusted $p=<0.01$; designated by $*$ ). By contrast, ventromedial neurons did not differ significantly in either absolute median firing rates (inset graph, Friedman two-way ANOVA, $p=0.2$ ) or in direct comparisons of the various behavioral states (horizontal boxes, one-sample $t$ test, $H_{0}=0$; Bonferroni adjusted $\left.p=>0.05\right)$.

In contrast to neurons in the dorsolateral region, firing rates in the ventromedial neostriatum did not differ significantly in either rate or variability across syntactic chain, other grooming, or behavioral resting epochs (Friedman two-way ANOVA, $p=0.2$; $n=27$ ). Overall, rate coding of grooming and resting states was coarse and weakly discriminated in the ventromedial striatum.

In addition, we also evaluated within individual neurons the effects of behavioral epoch, by computing the differences in median rates between each pair of epochs (chain vs rest, nonchain vs rest, and chain vs nonchain). In this way, each neuron served as its own control. For neurons in the ventromedial striatum, the distribution of rate differences did not differ across epochs (Fried- 

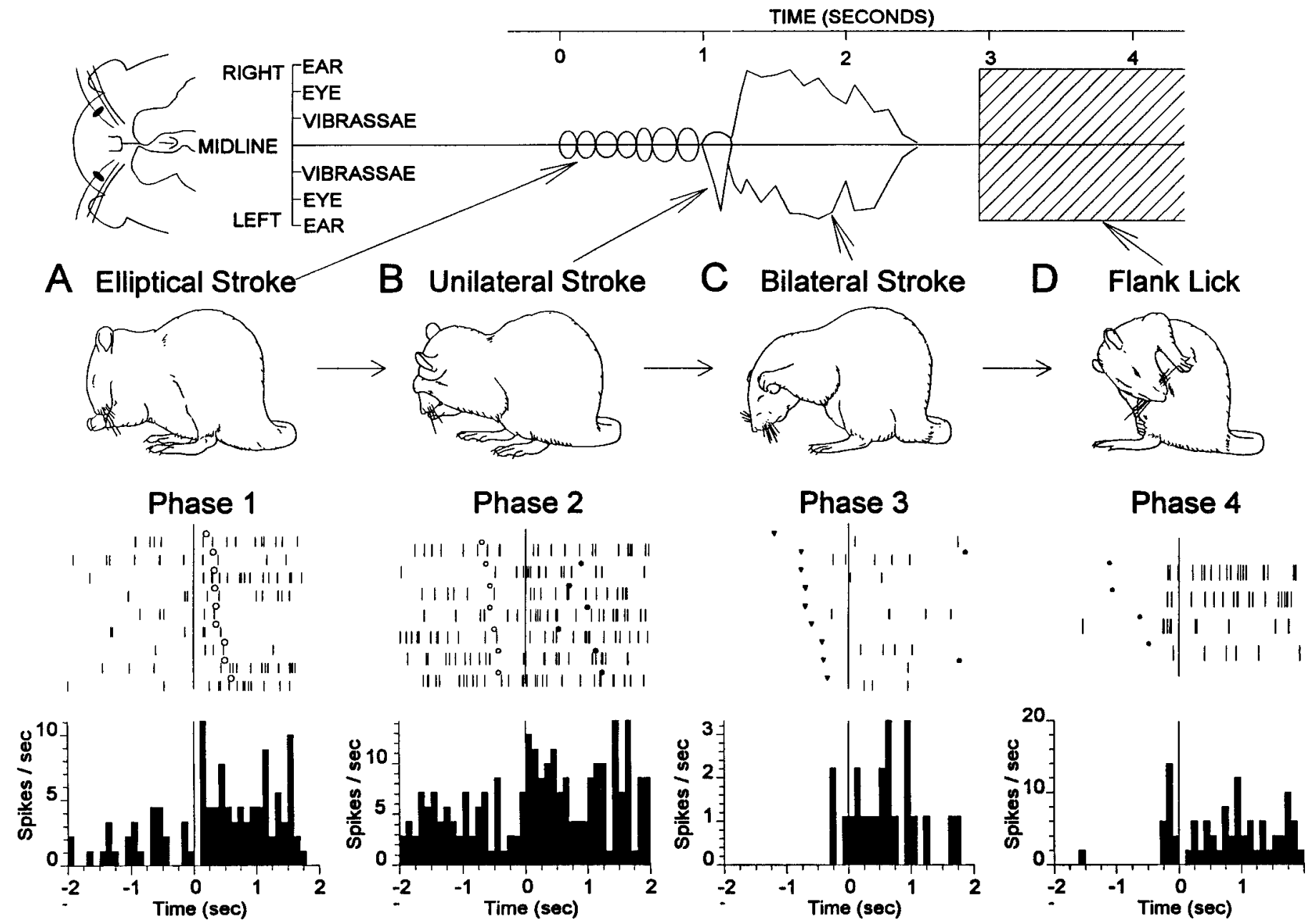

Figure 3. Neuronal coding of syntactic grooming phases. The schematic drawings $(A-D)$ show the four phases of syntactic grooming chains in the order they appear. A choreography diagram (top) illustrates the movement trajectory of the forelimbs as a function of time and distance from the midline (vertical dimension). The inset diagram to the left of the choreography diagram shows a rat's face as viewed from below on the video monitor on which the distances were determined. The excursions from the midline are measured from the midline to the center of the hand ( $Y$ dimension on this drawing) with the base of the vibrissae, eyes, and ears as landmarks. The bottom row of perievent time histograms and rasters from four different neurons illustrates neuronal activity changes of neurons that responded to a particular phase. Each example is a separate neuron in the dorsolateral striatum except $C$, which was recorded from ventromedial striatum. All four have increases in activity associated with the phase onset, which is at time $=0$ in each histogram and raster. The histogram represents the average firing rate ( $y$-axis) in bins $50 \mathrm{msec}$ wide. The marks in each spike train of the raster indicate the time in the spike train at which the preceding or following phase began. In $A$, the marks indicate phase 2 onset. In $B$, marks indicate phases 1 (time $<0$ ) and 3 (time $>0$ ). In $C$, marks indicate phases 2 (time $<0$ ) and 4 (time $>0$; note some are $>2 \mathrm{sec}$ and do not appear). In $D$, marks indicate phase 3 . The spike trains are sorted in the order of increasing phase 1 duration $(A, B)$, phase 2 duration $(C)$, and phase 3 duration $(D)$. Neuronal activity generally occurs at about the same time as movement onset except for the neuron in phase 4 , in which the change in activity precedes the onset of body licking.

man two-way ANOVA, $p=0.5$; Fig. 2), and the distributions were not significantly shifted from zero $\left(H_{0}=0\right.$; one-sample $t$ test, Bonferroni adjustment, $p>0.05$ each test; Fig. 2). In contrast, neurons in dorsolateral striatum had significantly faster firing rates during syntactic grooming chains compared both with nonchain grooming epochs and with behavioral rest epochs and faster rates during nonchain grooming epochs than during behavioral rest epochs (Friedman two-way ANOVA, $p<0.001$ in each case). Each distribution was significantly $>0$ (Bonferroni adjusted $p=$ 0.001, 0.009, and 0.001, respectively; Fig. 2).

\section{Phasic activation during grooming}

Much of the dynamic temporal information in individual neuron activity may be masked in the broad sweep of average firing rates obtained for epochs that last up to hundreds of seconds (syntactic chain, other grooming, and behavioral rest epochs). To uncover this dynamic information and compare the coding of syntactic chain sequences and nonchain grooming in more detail, we used perievent histogram analyses to examine phasic neuronal grooming responses (over periods ranging from $50 \mathrm{msec}$ up to $2 \mathrm{sec}$ around behavioral events). These analyses corroborated the special nature of grooming syntax. Individual neurons had activity changes strongly correlated to particular syntactic phases in a grooming chain (Fig. 3). Overall, more neurons in the striatum responded during syntactic grooming chains (41\%, 48 of 116) than during nonchain grooming $(14 \%, 16$ of $116 ; z$ test of proportions, $z=4.07$; $p<0.001)$. Despite the relatively rare occurrence and short duration of syntactic chains compared with other grooming, syntactic chains were the most effective at eliciting neuronal responses.

When syntactic chain and nonchain grooming categories were 
combined together, $51 \%$ (40 of 79) of the neurons in the dorsolateral site were responsive during some form of grooming compared with $38 \%$ (14 of 37) in the ventromedial site (Fig. 4); however, the anatomical site difference in overall responsiveness to grooming events were not significant $\left(\chi^{2}, p>0.05\right)$. Individual neurons were found at each site that responded to either syntactic chains, nonchain grooming, or both. The proportion that responded during syntactic chains was $46 \%$ (36 of 79) at the dorsolateral site, compared with $32 \%$ (12 of 37) at the ventromedial site $\left(\chi^{2}, p>0.05\right.$, although significant site differences existed in the coding of particular syntactic phases; see below). Only a small proportion of neurons (5\% in each region) responded only during nonchain grooming.

Both excitatory and inhibitory phasic responses were elicited during grooming. Excitatory responses were more common (99\% of neostriatal neurons) than inhibitory responses (20\% of neurons). Nearly all neurons that had inhibitory responses also had excitatory responses. Only one cell had an inhibitory response without an excitatory response. Typically, neuronal activity in the striatum was slow and irregular (Fig. 5), and chain-related activity was imposed on this ragged background. All of the neurons recorded in both regions had the type of irregular firing activity that is characteristic

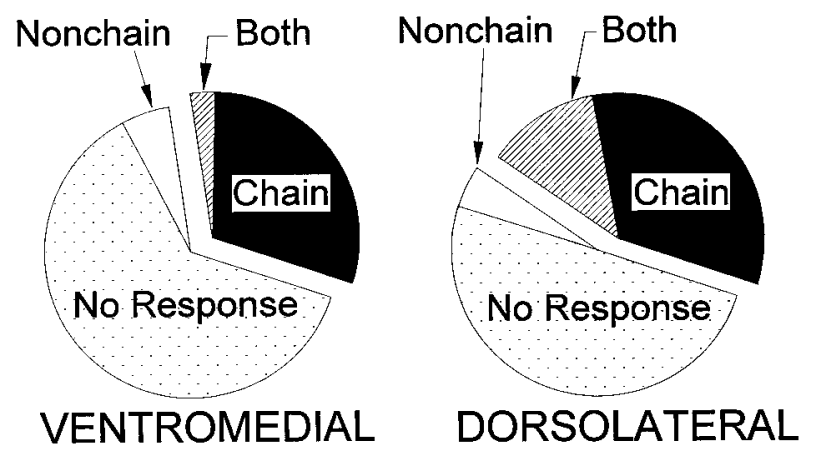

Figure 4. Proportion of grooming responses by neostriatal neurons. Ventromedial neurons are represented on the left $(n=37)$, and dorsolateral neurons are shown on the right $(n=79)$. The proportions of neurons that exhibited activity changes during chain grooming bouts are shown as the excised portions of the circles. A portion of these chainresponsive neurons also respond during nonchain grooming (crosshatching). There are more responsive neurons in the dorsolateral striatum overall and particularly, more neurons that responded to syntactic grooming chains. The relatively small proportions of neurons responding only during nonchain grooming are indicated by open hatching. Neurons with no response to grooming behavior are marked by dotted hatching. of medium spiny neostriatal neurons. None of the neurons had the more regular tonic discharge pattern of tonically active neurons (Aosaki et al., 1994).

\section{Postural movements}

Stepping, rearing, and other postural movements occur at high rates. Still, only a small proportion of neurons tested [8\% (3 of 36) dorsolateral neurons; $18 \%$ (4 of 22) ventromedial neurons] changed their activity in correlation to one of these movements. Of all spontaneous movements examined, syntactic grooming chains were the most potent phasic activator of neurons.

\section{Coding of syntactic phases}

Many neostriatal neurons responded distinctly to one or two particular phases of syntactic chains. This suggests that the neurons may code phase-specific properties of grooming syntax (Fig. $6)$. More than one-quarter of the neurons tested responded uniquely to just one syntactic phase [28\% (22 of 79) dorsolateral neurons; $27 \%$ (10 of 37 ) ventromedial neurons]. A smaller proportion of neurons showed multiphase responses, having activation during at least two syntactic phases. Of multiphase neurons responding to syntactic chains, the incidence of dorsolateral neurons outnumbered ventromedial neurons by a ratio of roughly $3: 1$ [18\% (14 of 79) dorsolateral neurons; 5\% (2 of 37) ventromedial neurons].

In general, neurons in the dorsolateral neostriatum were as likely to respond to multiple phases of a syntactic chain as to just a single phase, whereas neurons in the ventromedial striatum tended to respond to only a single phase of a syntactic chain $\left(\chi^{2}\right.$, $p<0.001)$. In most cases, multiphase neurons were active during consecutive syntactic grooming phases, although 29\% (4 of 14) of multiphase dorsolateral neostriatal neurons fired to syntactic phases that were separated by an intervening phase. The allocation of neurons to particular phases (Fig. 6), including both single-phase and multiphase neurons, ranged from $20 \%$ of dorsolateral neostriatal neurons responding during phase 1 (rapid ellipse strokes around the nose; 16 of 79 neurons) to $5 \%$ of ventromedial neostriatal neurons responding to phase 4 (flank licking; 3 of 37 neurons) and did not differ overall between the two striatal regions $\left(\chi^{2}, p>0.05\right)$.

\section{Intensity of phasic neuronal responses}

Syntactic coding was also reflected in the relative intensity of firing rate changes. Normalized values representing the magnitude of changes in firing rates were determined for every perievent his-
Phase 1, Elliptical Stroke

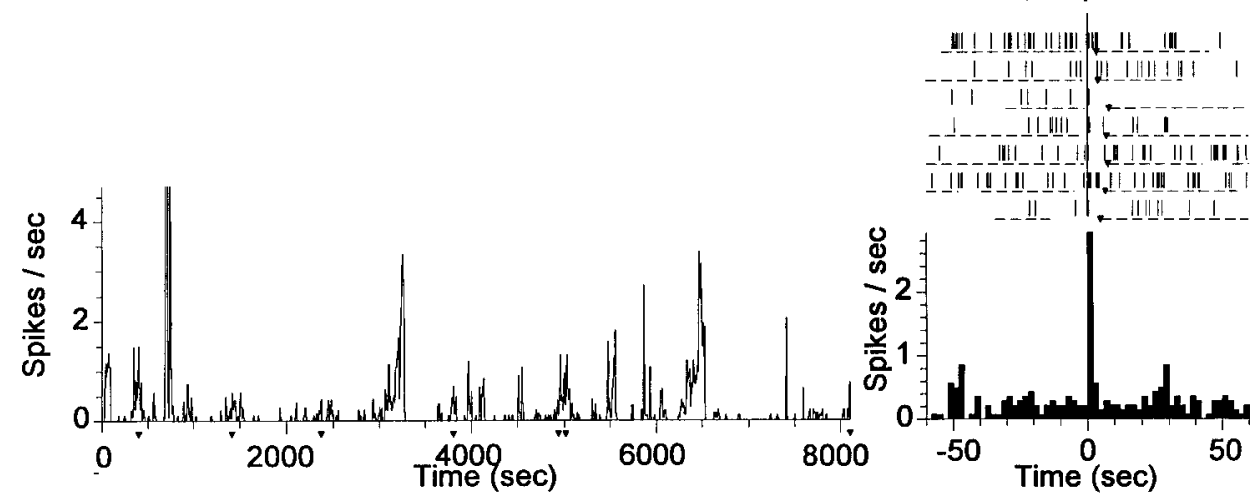

Figure 5. Sample dorsolateral neuron during entire recording session. The rate meter graph ( $5 \mathrm{sec}$ bin) on the left demonstrates the slow and irregular firing pattern of a single neuron in the dorsolateral neostriatum, typical of striatal neurons, recorded over $\sim 8000$ $\sec (2.2 \mathrm{hr})$. During this time seven syntactic chains occurred and are indicated by triangles under the $x$-axis. A perievent histogram aligned to the onset of the same seven syntactic chains (right, displays $\sim 1$ min periods) shows the neuron is still dominated by chainrelated activity when examined in a more fine-grained analysis. The filled triangles on each raster line indicate the end of the grooming chain. The dashed lines under each raster line indicate periods of nonchain grooming that preceded or followed the chain. 



Figure 6. Proportion of neurons that coded particular syntactic phases of grooming chains. Left, The proportions of responsive neurons (expressed as a percentage of total neurons tested on $y$-axis) are shown for each phase of the syntactic chain. Dorsolateral neurons were more responsive in every phase in comparison to ventromedial neurons. Right, Multiphase versus single-phase neurons. Dorsolateral neurons were also more likely than ventromedial neurons to have responses during more than one phase of the chain. In contrast, most ventromedial neurons are more likely to have a response during only a single phase of the chain.

togram for all neurons in the sample (79 dorsolateral and 37 ventromedial neurons) in $300 \mathrm{msec}$ periods for each behavioral marker described above (syntactic chain phases and morphologically similar nonchain grooming actions). The changes in rate were expressed as a percentage increase or decrease relative to a baseline period from -2 to -1 sec before the chain began. In the dorsolateral neostriatum the firing rate increased by $116 \%$ during syntactic chains relative to a baseline period before the chain (averaged across all neurons and all phases). The strength of these phasic responses to syntactic grooming was larger than would have been expected based on averaged tonic firing across behavioral epochs. For example, the analogous tonic behavioral state comparison (see above) of chain versus nonchain epochs revealed only a $16 \%$ higher rate during chains. In contrast, the average magnitude of phasic firing rate changes within a syntactic chain exceeded $100 \%$ above the baseline level just before the chain. Thus, the phasic syntactic chain response pushed a dorsolateral neuron to a true peak in terms of firing rate, which was at least five times higher than averaged tonic activity. Once again, a special role in syntax coding for the dorsolateral region of the neostriatum was indicated by the fact that its increase of $116 \%$ was almost four times greater (ANOVA, $p<0.05$ ) than the average increase in the ventromedial region (30\%; Fig. 7).

There was considerable variation between both phases and neurons. This was highlighted, for example, by an increase in phase 1 of $>200 \%$ along with considerable associated variability, compared with magnitudes of much less for other phases (Fig. 7). Some neurons were unresponsive to a chain, whereas others that had had little or no activity in the period before the chain were activated vigorously during the chain (e.g., see Fig. 9, Elliptical Stroke; 0.25 spikes/sec before chain to 9.58 spikes/sec during phase 1 gives an increase of $3832 \%$ ). In dorsolateral neurons, all phases had marked increases in firing, and no phase evoked an overall response larger than other phases (aggregated across neurons as a population, although individual neurons were phase-specific) (ANOVA, $p=$ 0.373). In contrast, ventromedial neurons had large increases in phase 3 and phase 1 but either no change or a relative decrease in other syntactic phases (Fig. 7; ANOVA, $p<0.01$ ).

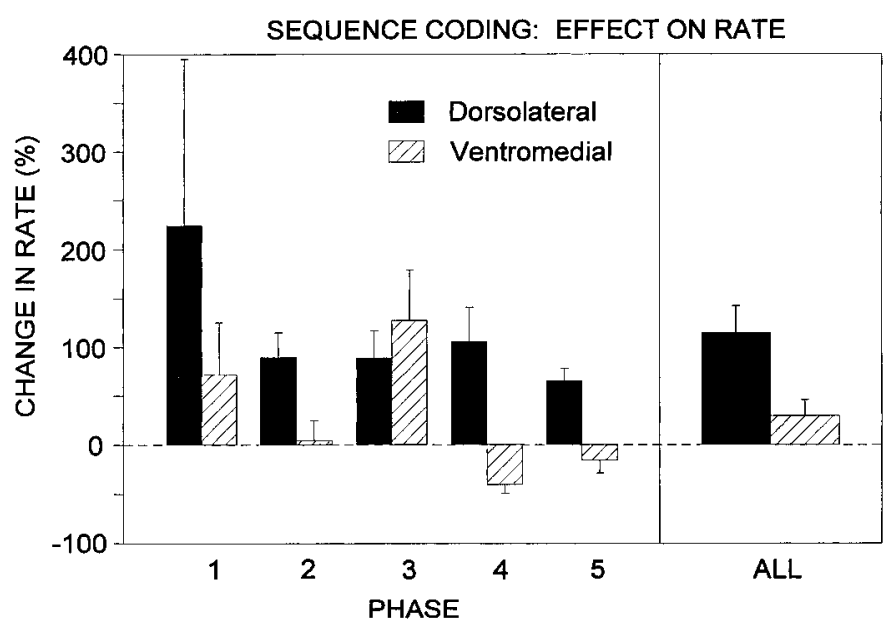

Figure 7. Phasic activity changes during grooming chains. The bars represent the average change in neuronal firing from the onset of the phase until $300 \mathrm{msec}$ after the onset, expressed as a percentage of the average activity in the period from -2 to $-1 \mathrm{sec}$ before the chain began. $\mathrm{SE}$ values are indicated on each bar. A value of zero (dashed baseline) indicates no change relative to the prechain period. Values $<0$ indicate a relative decrease in rate. Each bar represents one phase. Phase 5 represents the time period at which body licking ended. The graph on the right indicates the average for all five periods. Solid bars, Dorsolateral striatum; hatched bars, ventromedial striatum.

\section{Coding of movements versus sequence}

Neurons that responded to a syntactic chain phase were examined for their response during similar movements emitted during grooming outside the sequential context of syntactic chains. Body licking and forelimb stroke movements emitted outside of syntactic chains are morphologically similar to the corresponding chain movements - the primary difference between syntactic chain movements and other grooming movements is the pattern of serial order. Thus, a comparison of similar movements made in the different sequential contexts allows the dissociation of sequence properties from movement properties (motor activation or tactile or proprioceptive sensory feedback from the movement). Neurons related strictly to movement should respond in a similar way to a kinematically similar movement emitted in either sequential context. Conversely, neurons that code a sequential pattern should respond differently when morphologically similar movements are emitted in different sequential patterns. In fact, few neurons appeared to be movement-related by this criterion, and instead most chain-responding neurons coded a sequential pattern. Even in those cases in which chain and nonchain movements were kinematically most similar, such as phase 3 bilateral strokes in chains versus bilateral strokes that followed similar movement trajectories but were made as part of flexible nonchain grooming bouts, neostriatal neurons responded differentially to chain versus nonchain sequences (Fig. 8). We found that most neurons that responded during syntactic chain sequences failed to respond in the same way to similar movements made during nonchain grooming (Fig. 9). Only 16\% (6 of 36 responsive cells) of dorsolateral neurons that responded to syntactic chains had similar responses to equivalent movements during nonchain grooming in a manner that would allow them to be categorized as strictly "movement-related." One responsive neuron responded to the same strokes in chain and nonchain grooming, but the pattern of cell activity was different in the two contexts (Fig. 9, Flank Lick). None of the ventromedial neurons had similar chain 

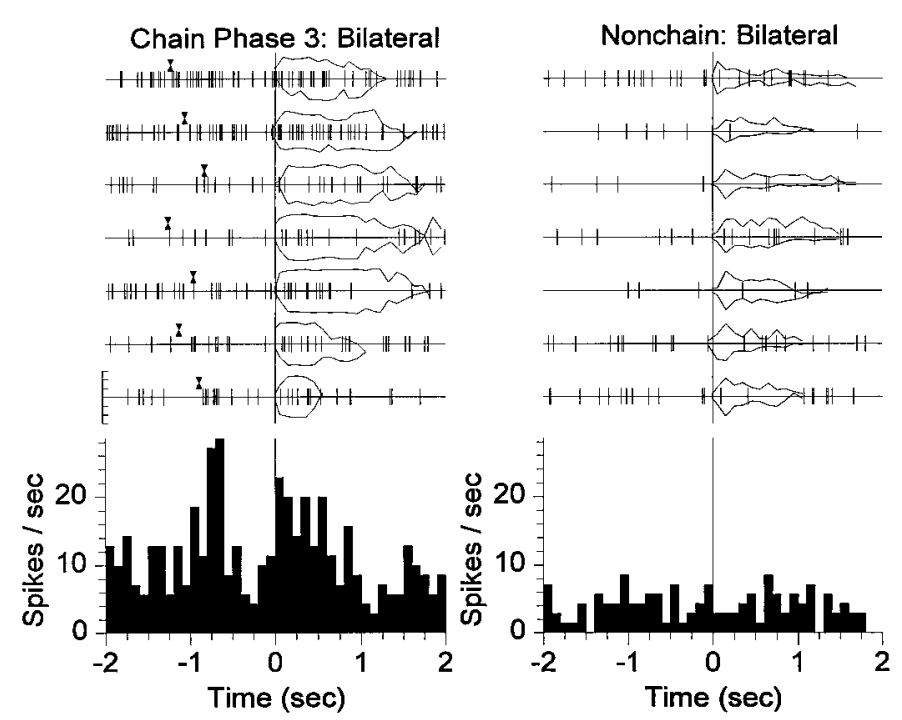

Figure 8. Neostriatal coding of syntax versus movement. This neuron in the dorsolateral striatum was activated during phase 3 (Bilateral strokes) of the syntactic grooming chain (left) but was not responsive to bilateral grooming strokes that were performed outside of the chain during sequentially flexible bouts of nonchain grooming (right). In other words, the neuron did not code the kinematic or dynamic properties of the bilateral strokes but rather was sensitive to features of these movements in the context unique to syntactic chains. The actual forelimb movements are shown by the choreographed trajectory representations superimposed on each spike train (format as in Fig. 3). The onset of the bilateral stroke, which is the alignment point for these spike trains and histogram, begins at time $=0$. The vertical axis to the left of the bottom trace in the raster on the left indicates the excursion dimensions in the same format as the one shown in Figure 3. Whereas the trajectories of nonchain grooming strokes were often smaller in amplitude, the two forepaws made similar movements over the face below the ears (in terms of stroke morphology, pattern, and time course) during syntactic chains and nonchain grooming. This particular neuron was also responsive during phase 1 of the chain, as indicated by the peak in the histogram at about $-0.8 \mathrm{sec}$. The marks above each raster line indicate the time at which the phase 1 strokes began.

and nonchain responses. The majority $(81 \%)$ of chain responsive neurons either had no response during nonchain grooming or a nonchain response to a stroke that was different from the chainevoked activity. Overall, the picture that emerged was one with a strong relationship of neostriatal activity to properties of sequence accompanied by a relatively weak relationship to movement properties per se.

The coding of sequence was characterized by a strong bias toward the sequential pattern of syntactic chains rather than to nonchain grooming sequences: only $5 \%$ (4 of 79) of dorsolateral neurons and $5 \%$ (2 of 37 ) of ventromedial neurons responded only to grooming movements made outside of the chain (and not to similar movements made within syntactic chains). In contrast, $33 \%$ (26 of 79 ) of dorsolateral neurons and $30 \%$ (11 of 37) of ventromedial neurons responded only to syntactic chains (and not during similar nonchain grooming movements occurring in different order). Only small proportions of dorsolateral neurons $(13 \%, 10$ of 79$)$ and ventromedial neurons (3\%, 1 of 37$)$ responded equivalently during both chain and nonchain categories of grooming movements (Fig. 4).

More neurons responded overall to syntactic grooming chains. To assess the quantitative pattern within phases, we determined the percentage of responses in each chain phase and nonchain equivalent in both striatal regions for all neurons ( $n=79$ dorso- lateral; $n=37$ ventromedial). Although phase 1 had a larger percentage of responses (ranging as high as $20.3 \%$ in dorsolateral striatum with an mean of $15.8 \%$ across all phases; Fig. 6), the overall distribution across phases was not significant (ANOVA, $p=0.938)$. The average response in equivalent strokes during nonchain grooming was much lower (an average of $7.3 \%$ across phases ranging up to a maximum of $12.7 \%$; Fig. 6 ). The type of grooming did matter, however, because neurons responded to chain grooming significantly more strongly than to nonchain grooming movements $(12.6 \pm 1.8$ vs $4.7 \pm 1.5 \%$ for chain and nonchain, respectively; ANOVA, $p<0.001)$. Additionally, the percentage of responses to the average phase of a syntactic chain in dorsolateral striatum was twice as high $(11.5 \%)$ as it was in the ventromedial region (5.7\%; ANOVA, $p<0.03)$.

\section{Timing of neuronal activity}

To address the question of whether the striatum might be initiating grooming actions or instead playing some role in the implementation of sequential motor actions, we examined the timing of neuronal activity related to the timing of movements. If the striatum is initiating the movements, it might be expected to have activity before the movements. The temporal relation between neuronal activity and behavior could be discerned most clearly for syntactic phase 1, although the onset of neuronal activity and of syntactic phase were both unambiguous. In these cases, neuronal activity almost always occurred during or after the onset of grooming movements rather than before them (Fig. 10). None of the dorsolateral neurons responsive to phase 1 of the syntactic chain had activity changes before the onset of the grooming strokes, and only one ventromedial neuron had an activity change before phase 1. By contrast, 15 of 79 dorsolateral neurons (19\%) and 5 of 37 ventromedial neurons (14\%) showed pronounced activity during phase 1 after the first elliptical stroke had begun. This temporal activation pattern for phase 1 suggests that striatal activity may a role in phase implementation rather than initiation.

Other syntactic phases exhibited timing relationships between neuronal activity and movement onset similar to the phase 1 temporal structure (Fig. 10). Only a small proportion of neuronal responses clearly preceded the onset of movement (Fig. 9, Flank Lick), and most neuronal responses occurred during or after the initial constituent of the phase. However, the classification of a midchain neuronal response as triggering or after the initiation of a particular phase is complicated by the rapid succession of syntactic chain phases. For example, neuronal activity that followed a phase 2 forelimb stroke could also be viewed instead as preceding a larger phase 3 stroke. Also, neostriatal neuronal activation usually persisted throughout several grooming movements within the phase and sometimes even longer than a single syntactic phase. Still, when pauses separated the movements, peak neuronal responses typically occurred after the movement had begun. Responses to grooming movements outside of the syntactic chain also usually coincided with or followed the movement onset (Fig. 10).

\section{DISCUSSION}

Our findings demonstrate that neuronal activity in rodent neostriatum is correlated to specific syntactic sequences of grooming movements. These neurons appeared to code the serial order of natural actions, and not the simple motor properties of constituent grooming movements, because their activity depended on the sequential relationship of movements to each other. Thus, it was 

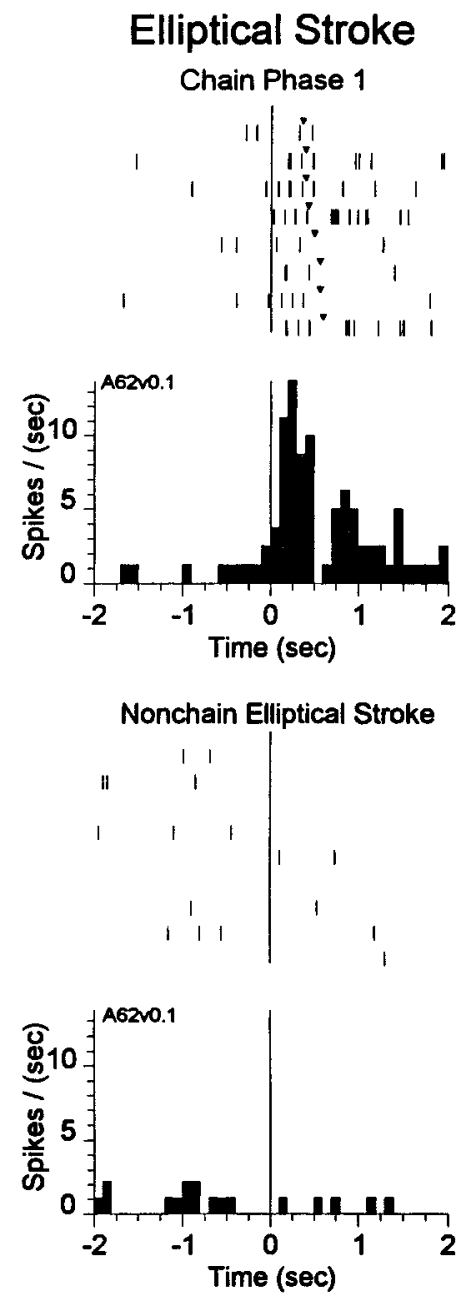

\section{Unilateral Stroke Chain Phase 2}

\section{Bilateral Stroke \\ Chain Phase 3}

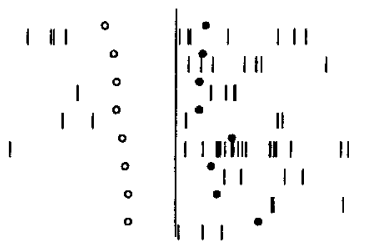

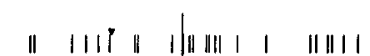

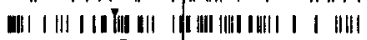

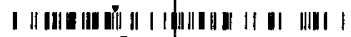

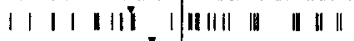

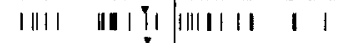

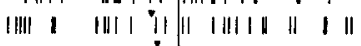

|| ||| | | || ||| | || || || || || || ||| | | | ||



Flank Lick

Chain Phase 4

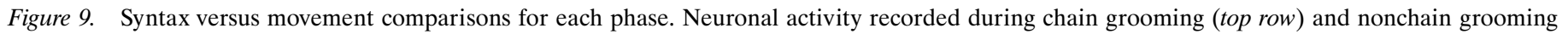

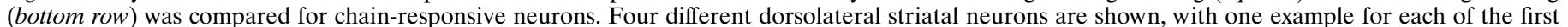

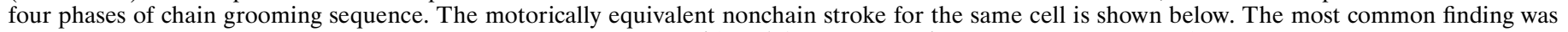

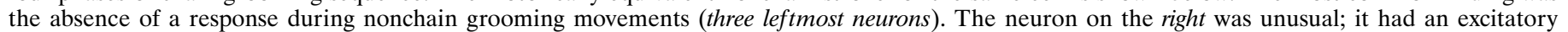

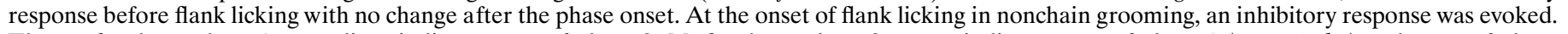

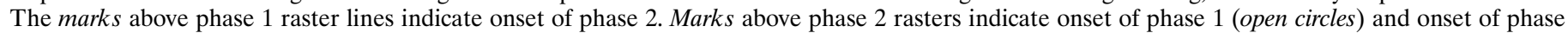

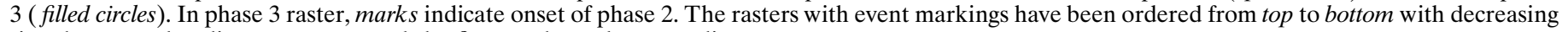
time between the alignment event and the first mark on the raster line.

the pattern of serial order, or action syntax, that was crucial for the activation of these neurons.

Neurons in the dorsolateral region of the neostriatum preferentially coded the serial order of movement compared with neurons in the ventromedial neostriatum. Although both regions had neurons that were sensitive to syntactic grooming sequences, dorsolateral neurons had larger increases in activity than ventromedial neurons during syntactic chains. Dorsolateral neurons were also more likely to respond during multiple phases of a syntactic grooming chain. This suggests that dorsolateral neurons may code syntactic patterns of movement serial order as a higherorder property, distributed over the duration of the chain. By contrast, activity of neurons in the ventromedial region actually declined during some phases of syntactic grooming chains, and ventromedial neurons were less likely to code either multiple phases or terminal phases. These findings suggest that the dorsolateral region may be concerned with syntactic phase-to-phase transitions or overall sequential structure, although ventromedial activity is concerned more simply with the onset of the chain.

Our conclusion that dorsolateral neostriatal neurons preferentially code grooming syntax is supported by the previous finding that lesions of the anterior dorsolateral neostriatum disrupt grooming syntax without disrupting grooming movements (Cromwell and Berridge, 1996). In contrast, lesions of other neostriatal regions, or of motor cortex, supplementary motor cortex, frontal cortex, cerebellum, etc., do not disrupt grooming syntax, even though several of those lesions do disrupt grooming movements (Berridge and Whishaw, 1992; Cromwell and Berridge, 1996). Similarly, grooming syntax emerges in ontogeny simultaneous with striatal maturation (Colonnese et al., 1996). The dorsolateral neostriatal neurons found in this study, which encode syntactic features of grooming patterns, may therefore contribute a causal function that is crucial to the behavioral implementation of grooming syntax. 

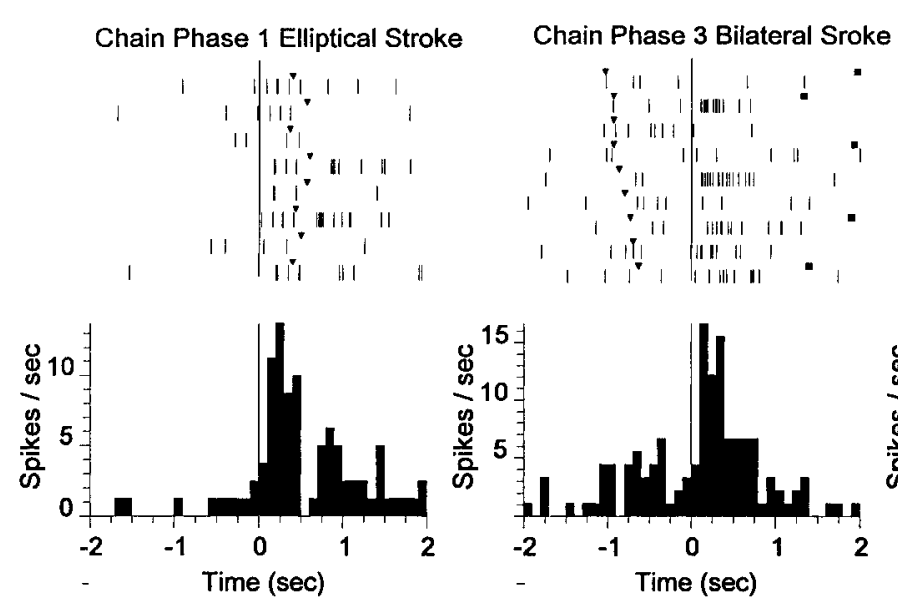

The principal type of syntax code identified by our study was the temporal pattern of spike activation (Aldridge and Gilman, 1991), which is probably meditated by patterned input to and intrinsic properties of medium spiny neurons (Wilson and Kawaguchi, 1996). Both the cerebral cortex and thalamus provide excitatory input to the striatum. Because an earlier study showed that cerebral cortical lesions fail to disrupt behavioral grooming syntax, it can be surmised that subcortical inputs may be of greatest importance for syntactic coding of grooming sequences (although future studies would be needed to confirm that hypothesis). Tonic changes of spike rate are another potential coding mechanism for sequences, but our results suggest that tonic coding within different behavioral states is weaker and coarser than short-term phasic rate changes.

The timing of neuronal activity relative to movement onset indicated that dorsolateral neostriatal activity probably does not either initiate the sequence or generate the syntactic pattern of serial order itself. Instead the neostriatum is more likely to be involved in implementing into behavior a syntactic signal generated elsewhere. A role in the implementation (rather than generation) of syntactic grooming sequences is consistent with the results of lesion and transection studies of the neural basis of behavioral grooming syntax. Elementary generation of the basic four-phase syntactic pattern can be performed by the isolated rodent pontine brainstem. Decerebrate rats, in which the brain has been transected either above the superior colliculus or above the pons and cerebellum, still generate occasional syntactic chain patterns of grooming more often than chance, even though they have marked deficits in sequence implementation (Berridge, 1989). That suggests the striatal role in grooming syntax may be to gate the translation of brainstem-generated syntactic patterns into behavior rather than to generate the pattern entirely itself.

This role is also compatible with recent models of the neostriatum (Graybiel, 1995; Graybiel and Kimura, 1995; Jackson and Houghton, 1995; Gabrieli, 1996). A recent review points out that several computational models of basal ganglia "have emphasized pattern recognition or mutual competition, or a combination of the two, to form pattern classification networks" (Beiser et al., 1997). Regarding grooming syntax, such networks might code or recognize the occurrence of a syntactic chain signal from the brainstem and dynamically potentiate its access to sensorimotor
Figure 10. Timing of neuronal activity. The timing relationships of neuronal activity to the onset of movement is shown for three dorsolateral neurons. The format of each perievent histogram is simiNonchain Large Stroke (right) lar, with the onset of the first movement in $\|$ | \|\|$\|111\| \|$ the phase aligned to time $=0$ on the

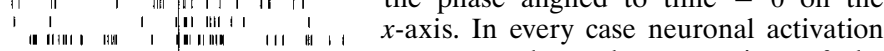
InIII I I I Initin Ine

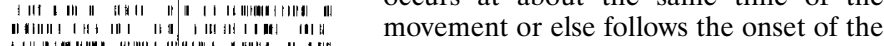

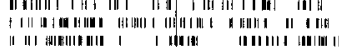

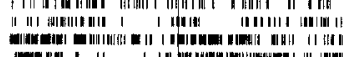

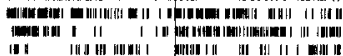

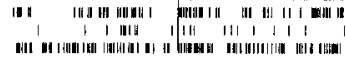
movement. Left, A neuron activated during phase 1 of the chain (Elliptical strokes) is shown. The marks in each spike train after time $=0$ indicate the onset time of phase 2. Center, Another neuron responsive to the onset of phase 3 (Bilateral stroke). The marks before the time 0 axis indicate the onset of phase 2. The marks after time 0 (where visible) indicate the onset of phase 4. Right, A different dorsolateral neuron illustrates a similar timing relationship to a nonchain large unilateral stroke with the right limb (recording on left side of striatum). output mechanisms that directly control movement, although suppressing competing signals. Although such a causal role is suggested for neurons in the anterior dorsolateral neostriatum, the role of ventromedial neurons, in which lesions do not disrupt behavioral grooming syntax, is less clear. However, ventromedial neurons might provide feedback by monitoring the progress of a sequential pattern or might modulate functions that integrate syntactic grooming chains with other aspects of behavior. At present, these hypotheses are only speculative, and additional study will be needed to fully characterize the roles that different neostriatal regions play in behavioral sequencing.

A distinctive feature of the movement sequence studied here is that the serial pattern is "instinctive" or species-typical. A similar four-phase pattern is emitted naturally without training or memorization by rats and by other species from all suborders of Rodentia. The ubiquity of the grooming syntax pattern among rodents suggests that it probably evolved at least 60 million years ago before rodent suborders and species diverged (Berridge, 1990). Neostriatal specializations for sequencing behavior are therefore likely to be ancient and may have evolved originally to coordinate instinctive movement sequences. This is consistent with conclusions that striatal circuits are involved in the sequential patterning of other behavior, such as birdsong, in nonmammalian vertebrates (Margoliash et al., 1994; Yu and Margoliash, 1996). The conclusion that basal ganglia circuits implement the sequential order of species-typical behavior is also supported by the report that instinctive sequences of movements used in play by rats are disrupted by lesions that destroy neostriatal dopamine projections (Pellis et al., 1992).

What is the relationship between the putative original role of basal ganglia in species-typical behavioral sequences and the roles of modern human and primate basal ganglia in learned behavioral sequences and in cognitive sequences? The striatal circuitry that evolved originally to coordinate stereotyped innate sequences of movements would also have utility as a preadaptation for neural mechanisms of behavioral sequencing. The evolution of the neostriatum may have subsumed preexisting circuitry for instinctive movement sequences, and incorporated additional cortical connections, to extend sequencing functions to more highly elaborated forms of rule-driven behavior. The striatum and basal ganglia are clearly activated by learned movements in primates 
(Aldridge et al., 1980a,b; DeLong and Georgopoulos, 1981; Alexander and Crutcher, 1990; Kimura, 1990), including sequential patterns of movements (Kermadi et al., 1993; Kermadi and Joseph, 1995; Mushiake and Strick, 1995).

Neostriatal coding of learned sequences of movements is consistent with suggestions that the basal ganglia in humans may be a repository for nondeclarative memories or motor habits (Mishkin et al., 1984; Knowlton et al., 1996). It is of interest that neurons in prefrontal cortex also appear to participate in coding learned behavioral sequences in monkeys (Barone and Joseph, 1989), whereas rodent grooming syntax appears less dependent on neocortex (Berridge and Whishaw, 1992). This difference between primates and rodents suggests that neural circuits for behavioral sequencing may have been encephalized during primate evolution, to incorporate additional cortical structures for sequencing more elaborate or abstract forms of behavior.

A culmination of this trend toward the sequential control of increasingly abstract behavioral elements may be revealed in some of the consequences of basal ganglia pathology in humans. For example, Parkinson's patients been suggested to have special difficulty in performing sequences of voluntary movements above and beyond their other deficits, for example, in executing sequential switches among different hand movements, and even in recognizing movement sequences performed by others (Harrington and Haaland, 1991). Huntington's patients have similarly been found to have special deficits on sequential movement tasks that involve the use of advance information to guide later movements (Georgiou et al., 1994), and also "ideomotor" deficits that relate a pattern of movement to a cognitive concept (e.g., tool use) (Shelton and Knopman, 1991).

At the most abstract level, the neostriatum has even been suggested to be involved in sequences of human language and sequences of thought. Specific deficits in language syntax, both for production and comprehension, appear to accompany a set of striatal lesions in humans (Brunner et al., 1982; Damasio and Damasio, 1992; Volkmann et al., 1992). The pathological repetitions of spoken words in Tourette's syndrome (Cummings and Frankel, 1985) and the tormenting habits and thoughts of obsessive-compulsive disorder (Rapoport and Wise, 1988), both of which may be associated with pathology of the basal ganglia, have been argued to reflect basal ganglia participation in sequencing the highest of cognitive processes. As Marsden (1984) provocatively suggested, "the sequencing of motor action and the sequencing of thought could be a uniform function performed by the basal ganglia." Our study indicates that the kernel of basal ganglia sequencing functions may lie in controlling basic syntactic sequences of mammalian motor behavior, such as rodent grooming syntax.

\section{REFERENCES}

Aldridge JW, Gilman S (1991) The temporal structure of spike trains in the primate basal ganglia-afferent regulation of bursting demonstrated with precentral cerebral cortical ablation. Brain Res 543:123-138.

Aldridge JW, Anderson RJ, Murphy JT (1980a) Sensory motor processing in the caudate nucleus and globus pallidus: a single unit study in behaving primates. Can J Physiol Pharmacol 58:1192-1201.

Aldridge JW, Anderson RJ, Murphy JT (1980b) The role of the basal ganglia in controlling a movement initiated by a visually presented cue. Brain Res 192:3-16.

Aldridge JW, Berridge KC, Herman M, Zimmer L (1993) Neuronal coding of serial order: syntax of grooming in the neostriatum. Psychol Sci 4:391-395.
Alexander GE, Crutcher MD (1990) Preparation for movement: neural representations of intended direction in 3 motor areas of the monkey. J Neurophysiol 64:133-150.

Aosaki T, Tsubokawa H, Ishida A, Watanabe K, Graybiel AM, Kimura M (1994) Responses of tonically active neurons in the primate's striatum undergo systematic changes during behavioral sensorimotor conditioning. J Neurosci 14:3969-3984.

Barone P, Joseph JP (1989) Prefrontal cortex and spatial sequencing in macaque monkey. Exp Brain Res 78:447-464.

Beiser DG, Hua SE, Houk JC (1997) Network models of the basal ganglia. Curr Opin Neurobiol 7:185-190.

Berridge KC (1989) Progressive degradation of serial grooming chains by descending decerebration. Behav Brain Res 33:241-253.

Berridge KC (1990) Comparative fine structure of action: rules of form and sequence in the grooming patterns of six rodent species. Behaviour 113:21-56.

Berridge KC, Fentress JC (1986) Contextual control of trigeminal sensorimotor function. J Neurosci 9:325-330.

Berridge KC, Fentress JC (1987) Disruption of natural grooming chains after striatopallidal lesions. Psychobiology 15:336-342.

Berridge KC, Whishaw IQ (1992) Cortex, striatum, and cerebellum: control of serial order in a grooming sequence. Exp Brain Res 90:275-290.

Berridge KC, Fentress JC, Parr H (1987) Natural syntax rules control action sequence of rats. Behav Brain Res 23:59-68.

Brunner RJ, Kornhuber HH, Seemuller E, Suger G, Wallesch CW (1982) Basal ganglia participation in language pathology. Brain Lang 16:281-299.

Colonnese MT, Stallman EL, Berridge KC (1996) Ontogeny of action syntax in altricial and precocial rodents: grooming sequences of rat and guinea pig pups. Behaviour 113:1165-1195.

Cromwell HC, Berridge KC (1996) Implementation of action sequences by a neostriatal site: a lesion mapping study of grooming syntax. J Neurosci 16:3444-3458.

Cummings JL, Frankel M (1985) Gilles de la Tourette syndrome and the neurological basis of obsessions and compulsions. Biol Psychiatry 20:117-126.

Damasio AR, Damasio H (1992) Brain and language. Sci Am 267:88-95.

DeLong MR, Georgopoulos AP (1981) Motor functions of the basal ganglia. In: Handbook of physiology, Sec 1, Nervous system, Vol 2, Motor control (Brooks V ed), pp 1017-1061. Baltimore: Williams \& Wilkins.

Fentress JC (1972) Development and patterning of movement sequences in inbred mice. In: The Biology of behavior (Kiger K ed), pp 83-132. Corvallis, OR: Oregon State University.

Gabrieli J (1996) Contribution of the basal ganglia to skill learning and working memory in humans. In: Models of information processing in the basal ganglia (Houk JA, Davis JL, Beiser DB, eds), pp 277-294. Cambridge, MA: MIT.

Georgiou N, Bradshaw JL, Iansek R, Phillips JG, Mattingley JB, Bradshaw JA (1994) Reduction in external cues and movement sequencing in Parkinson's disease. J Neurol Neurosurg Psychiatry 57:368-370.

Graybiel AM (1995) Building action repertoires: memory and learning functions of the basal ganglia. Curr Opin Neurobiol 5:733-741.

Graybiel AM, Kimura M (1995) Adaptive neural networks in the basal ganglia. In: Models of information processing in the basal ganglia (Houk JA, Davis JL, Beiser DB, eds), pp 103-116. Cambridge, MA: MIT.

Harrington DL, Haaland KY (1991) Sequencing in Parkinson's disease-abnormalities in programming and controlling movement. Brain 114:99-115.

Jackson S, Houghton G (1995) Sensiormotor selection and the basal ganglia. In: Models of information processing in the basal ganglia (Houk JA, Davis JL, Beiser DB, eds), pp 337-369. Cambridge, MA: MIT.

Jaeger D, Gilman S, Aldridge JW (1990) A multiwire microelectrode for single unit recording in deep brain structures. J Neurosci Methods 32:143-148.

Kermadi I, Joseph JP (1995) Activity in the caudate nucleus of monkey during spatial sequencing. J Neurophysiol 74:911-933.

Kermadi I, Jurquet Y, Arzi M, Joseph JP (1993) Neural activity in the caudate nucleus of monkeys during spatial sequencing. Exp Brain Res 94:352-356.

Kimura M (1990) Behaviorally contingent property of movementrelated activity of the primate putamen. J Neurophysiol 63:1277-1296. 
Knowlton BJ, Mangels JA, and Squire LR (1996) A neostriatal habit learning system in humans. Science 273:1399-1402.

Lashley KS (1951) The problem of serial order in behavior. In: Cerebral mechanisms in behavior (Jeffress LA, ed), pp 112-146. New York: Wiley. Margoliash D, Fortune ES, Sutter ML, Yu AC, Wren-Hardin BD, Dave A (1994) Distributed representation in the song system of oscines: evolutionary implications and functional consequences. Brain Behav Evol 44:247-264.

Marsden CD (1984) Which motor disorder in Parkinson's disease indicates the true motor function of the basal ganglia? In: Functions of the basal ganglia. Ciba Foundation Symposium 107, pp 225-241. London: Pitman.

Mishkin M, Malamut B, Bachevalier J (1984) Memories and habits: two neural systems. In: Neurobiology of human learning and memory (Lynch G, McGaugh JL, Wienberger NM, eds), pp 65-77. Guilford.

Miyachi S, Hikosaka O, Miyashita K, Karadi Z, Rand MK (1997) Differential roles of monkey striatum in learning of sequential hand movement. Exp Brain Res 115:1-5.

Mushiake H, Strick PL (1995) Pallidal neuron activity during sequential arm movements. J Neurophysiol 74:2754-2758.
Pellis SM, Pellis VC, Whishaw IQ (1992) The role of the cortex in play fighting by rats: developmental and evolutionary implications. Brain Behav Evol 39:270-284.

Rapoport JL, Wise SP (1988) Obsessive-compulsive disorder: evidence for basal ganglia dysfunction. Psychopharmacol Bull 24:380-384.

Richmond G, Sachs BD (1978) Grooming in Norway rats: the development and adult expression of a complex motor pattern. Behaviour 75:82-96.

Shelton PA, Knopman DS (1991) Ideomotor apraxia in Huntington's disease. Arch Neurol 48:35-41.

Swanson LW (1992) Brain maps: structure of the rat brain. Amsterdam: Elsevier.

Volkmann J, Hefter H, Lange HW, Freund HJ (1992) Impairment of temporal organization of speech in basal ganglia diseases. Brain Lang 43:386-399.

Wilson CJ, Kawaguchi Y (1996) The origins of two-state spontaneous membrane potential fluctuations of neostriatal spiny neurons. J Neurosci 16:2397-2410.

Yu AC, Margoliash D (1996) Temporal hierarchical control of singing in birds. Science 273:1871-1875. 